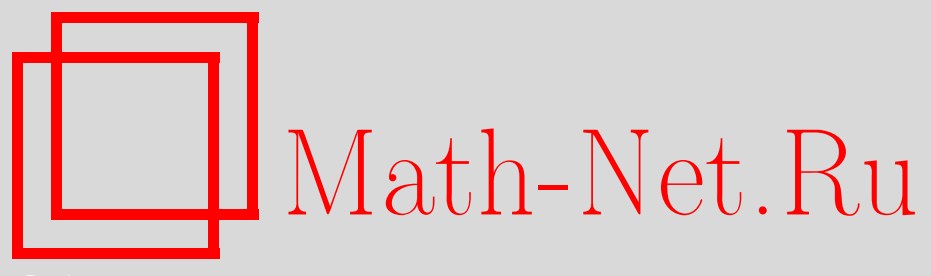

Б. А. Амосов, В. М. Бухштабер, М. М. Маламуд, С. П. Новиков, Г. В. Розенблюм, Т. А. Суслина, Л. Ф. Фридлендер, А. А. Шкаликов, Михаил Семенович Агранович (некролог), УМН, 2018, том 73, выпуск $1,173-178$

DOI: https://doi.org/10.4213/rm9815

Использование Общероссийского математического портала Math-Net.Ru подразумевает, что вы прочитали и согласны с пользовательским соглашением http://www.mathnet.ru/rus/agreement

Параметры загрузки:

IP : 54.81 .137 .203

26 апреля 2023 г., 11:05:49

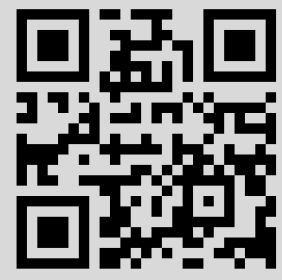




\section{Михаил Семенович Агранович}

14 февраля 2017 г. ушел из жизни Михаил Семенович Агранович, выдающийся математик, специалист в области дифференциальных уравнений и спектральной теории операторов.

Михаил Семенович родился 4 января 1931 г. в Москве. Он воспитывался матерью Евгенией Абрамовной, которую очень любил (родители разошлись, когда он был ребенком). Во время войны Михаил с матерью были в эвакуации в Саратове.

В 1948 г. он поступил на механико-математический факультет Московского государственного университета (МГУ им. М.В. Ломоносова), а в 1953 г. окончил его. В студенческие годы Михаил занимался под руководством Д. Е. Меньшова, изучая методы суммирования рядов. По этой теме ему удалось получить впечатляющие результаты. В частности, он дал отрицательный ответ на поставленный В. М. Даревским вопрос о совместности регулярных транслирующих ме-

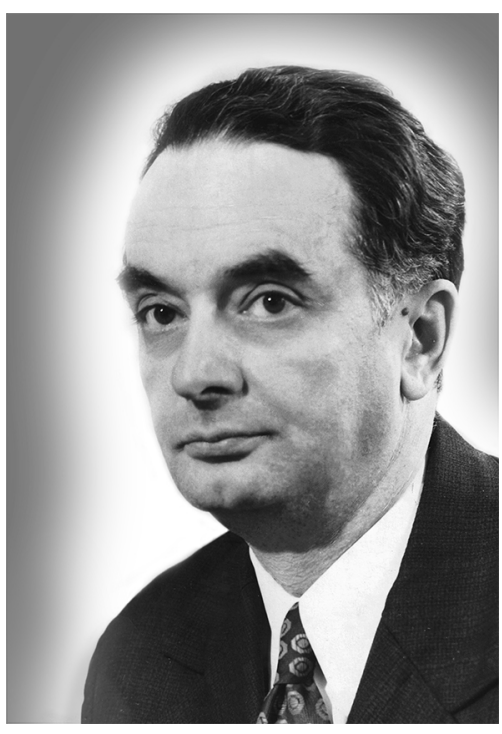
тодов суммирования и получил положительный ответ на вопрос Д. Е. Меньшова о совместности регулярных нормальных вполне транслирующих методов суммирования. Эта его первая объемная студенческая работа была опубликована в 1954 г. в журнале "Ученые записки МГУ" .

После окончания МГУ Михаил Семенович работал в математической редакции издательства "Иностранная литература" и одновременно учился в заочной аспирантуре. В 1959 г. под руководством Г. Е. Шилова он защитил кандидатскую диссертацию, посвященную дифференциальным операторам $P(D)$ общего вида с постоянными коэффициентами (во всем пространстве $\mathbb{R}^{n}$ или в ограниченной области $\Omega \subset \mathbb{R}^{n}$ ). Изучались вопросы разрешимости и свойства решений уравнения $P(D) u=f$ в различных классах обобщенных функций.

В 1960 г. Михаил Семенович перешел на преподавательскую работу - сначала во Всесоюзный заочный машиностроительный институт, а вскоре после этого в Московский институт электроники и математики (МИЭМ). В этом институте (позднее влившемся в Высшую школу экономики) Михаил Семенович работал до конца своей жизни. В 1992-1998 гг. он заведовал кафедрой математического анализа в МИЭМ.

В 1962 г. М. С. Агранович опубликовал статью об индексе эллиптического оператора. Это произошло после публикации важной статьи И. М. Гельфанда "Об эллиптических уравнениях" (УМH, 15:3(93) (1960), 121-132), где общая проблема индекса была сформулирована (примечательно, что автор благодарит М. С. Аграновича

DOI: https://doi.org/10.4213/rm9815 
“за большую помощь при подготовке статьи"). Эта тема вскоре получила существенное развитие в совместной работе Михаила Семеновича с А. С. Дыниным, где были получены формулы, связывающие индексы различных эллиптических краевых задач (формулы Аграновича-Дынина). Согласно одной из этих формул, разность индексов двух эллиптических операторов, заданных одним и тем же дифференциальным выражением с разными краевыми условиями, равна индексу некоторого эллиптического псевдодифференциального оператора (ПДО) на границе области (его главный символ выражается через матрицы Шапиро-Лопатинского соответствующих краевых задач). Согласно другой формуле, разность индексов двух эллиптических операторов с коэффициентами, совпадающими на границе области, и с общими краевыми условиями равна индексу некоторого эллиптического ПДО на многообразии, полученном склейкой вдоль границы двух экземпляров области. Эти формулы стали важным этапом в развитии теории индекса и позднее обобщались на другие ситуации.

Широко известна фундаментальная формула Атьи и Зингера для индекса эллиптического дифференциального оператора на компактном ориентированном многообразии. М.Ф. Атья и И. М. Зингер во введении к своей статье "The index of elliptical operators on compact manifolds" (Bull. Amer. Math. Soc., 69:3 (1963), 422-433), написали (мы сохраняем нумерацию цитирований в этой статье):

"In his paper [16] Gelfand posed the general problem of investigating the relationship between topological and analytical invariants of elliptic differential operators. In particular he suggested that it should be possible to express the index of an elliptic operator in topological terms. This problem has been taken up by Agranovich [2, 3], Dynin [3, 14, 15], Seeley [20, 21] and Vol'pert [22], ${ }^{1}$ who have solved it in special cases".

Заканчивается эта статья так:

"The problem of extending Theorem 1 (знаменитая формула Атьи и Зингера - прим. ред.) to manifolds with boundary presents some difficulties. However, using the results of [3] (статья Аграновича и Дынина прим. ред.) one can use Theorem 1 to compute the index of a large class of boundary problems". ${ }^{2}$

В 1962-1964 гг. М.С. Агранович принимал активное участие в разработке общей теории краевых (и начально-краевых) задач для эллиптических, параболических и гиперболических уравнений с гладкими коэффициентами. Вместе с М. И. Вишиком он изучал эллиптические задачи, полиномиально зависящие от параметра. Ими была доказана однозначная разрешимость таких задач при больших значениях параметра

\footnotetext{
${ }^{1}$ Помимо упомянутой работы [22], вышедшей в 1962 г., А. И. Вольперт имел более раннюю работу "Об индексе и нормальной разрешимости граничных задач для эллиптических систем дифференциальных уравнений на плоскости" ( $T p . M M O, 10$ (1961), 41-88), которая была написана (как сообщается в статье) на основе результатов, представленных автором на заседании Московского математического общества в 1960 г. - Прим. ред.

${ }^{2}$ Понятие индекса сингулярных скалярных интегральных операторов на окружности с конечномерными ядром и коядром ввел Фриц Нётер (см. F. Noether, "Über eine Klasse singulärer Integralgleichungen", Math. Ann., 82 (1920), 42-63). Ненулевой индекс нарушает альтернативу Фредгольма. В СССР теория сингулярных интегральных уравнений с ненулевым индексом получила интенсивное развитие в школе Н. И. Мусхелишвили в Грузии (см. С. П. Новиков, "Топология в XX веке: взгляд изнутри", УМH, 59:5(359) (2004), 3-28). Уравнения и операторы с ненулевым индексом были названы нётеровыми. Позже их стали чаще называть фредгольмовыми.

В 1934 г. Фриц Нётер, сын Макса Нётера и брат Эмми Нётер, эмигрировал как еврей и коммунист из Германии в СССР. Он стал профессором Томского университета. В 1937 г. Ф. Нётер был репрессирован и до сентября 1941 г. находился в тюрьме г. Орёл. Дальнейшие сведения о судьбе Ф. Нётера противоречивы (см. http:/litbook.ru/article/7746/). В 1988 г. Верховный суд СССР реабилитировал его полностью.
} 
и были установлены оценки решения в нормах, содержащих параметр. Их результаты стали классическими и ныне широко известны как “теория Аграновича-Вишика". Эти результаты были применены и к доказательству однозначной разрешимости общих параболических начально-краевых задач в цилиндрических областях. Техника эллиптических задач с параметром оказалась также ключевой в доказательстве теоремы об аналитическом продолжении дзета-функции эллиптического оператора, полученном Р. Сили несколькими годами позже.

Михаил Семенович был одним из пионеров в становлении теории псевдодифференциальных операторов. Значительное влияние на развитие этой теории оказали его работы по эллиптическим сингулярным интегральным операторам, выполненные им в 1963-1965 гг., еще до появления работ К. Фридрихса, П. Лакса, Дж. Кона, Л. Ниренберга и Л. Хёрмандера, после которых термин "псевдодифференциальный оператор" стал общепринятым вместо терминов "сингулярный интегральный оператор" или "оператор Кальдерона-Зигмунда". Изучение граничных задач для ПДО и систем ПДО было приоритетным направлением исследований Михаила Семеновича вплоть до начала 1970-х годов.

В 1966 г. М. С. Агранович защитил докторскую диссертацию, в которую вошли результаты по теории индекса, по эллиптическим задачам с параметром и эллиптическим сингулярным операторам. В 1971 г. он стал профессором.

В 1970-х годах научные интересы Михаила Семеновича смещаются в сторону спектральной теории операторов, в первую очередь несамосопряженных. Толчком к этому стало сотрудничество с группой физиков под руководством Б. З. Каценеленбаума. В теории дифракции встречаются задачи, где спектральный параметр входит в граничное условие либо в условие сопряжения на поверхности Г. При сведении этих задач на Г естественным образом возникают спектральные задачи для интегральных или интегро-дифференциальных операторов на $Г$, как правило несамосопряженных. Имевшиеся тогда методы теории несамосопряженных операторов оказались недостаточными для адекватного решения возникающих задач. Михаил Семенович существенно развил спектральную теорию несамосопряженных операторов.

Для операторов $A$, близких к самосопряженным, обычно предполагалось, что $A=S+B$, где $S$ - самосопряженный оператор с дискретным спектром, а $B$ подчинен некоторой степени $S^{q}, 0 \leqslant q<1$, оператора $S$. Михаил Семенович столкнулся со случаями, когда $q$ может быть отрицательным и даже равным $-\infty$. Для таких операторов им были установлены более точные варианты теорем о сходимости "рядов Фурье со скобками" по корневым векторам и получены неулучшаемые оценки скорости сходимости.

Работая с конкретными операторами, М. С. Агранович пришел к пониманию, что теоремы о базисности корневых векторов несамосопряженных операторов удобнее иметь для операторов вида $A=S+B$, где $S$ самосопряжен, а $B$ - возмущение, подчиненное оператору $S$ в смысле квадратичных форм с порядком $q<1$. Для таких операторов он доказал теоремы о безусловной базисности корневых векторов при условии $p^{-1} \leqslant 1-q$, где $p$ - порядок оператора $S$. В случае, когда последнее условие не выполняется, он доказал теоремы о базисности корневых векторов для суммирования методом Абеля-Лидского.

В 1980-х годах М. С. Агранович исследовал спектральные свойства эллиптических с параметром ПДО на замкнутом многообразии. Здесь, в частности, были найдены асимптотические характеристики распределения собственных значений эллиптических операторов, далеких от самосопряженных. В этой ситуации собственные значения не приближаются к некоторому выделенному направлению в комплексной области (что типично для операторов, близких к самосопряженным), а располагаются вдоль всех направлений в некотором угле. Другая важная тема этого периода анализ эллиптических ПДО на замкнутой кривой, в частности таких, к которым сводятся спектральные граничные задачи в двумерных областях. Далеко развивая идеи 
Г. В. Розенблюма, Михаил Семенович построил теорию таких операторов, опирающуюся на ряды Фурье, а не на традиционно используемое преобразование Фурье. На этом пути для широкого класса ПДО на замкнутой кривой было найдено преобразование к оператору с постоянным символом $P(D)$, с погрешностью, подчиненной любой заранее заданной степени оператора $P(D)$. Последующее применение абстрактных результатов о возмущениях привело к многочленным асимптотическим формулам для собственных значений, а также теоремам о сходимости и суммируемости спектральных разложений.

С середины 1990-х годов основные интересы Михаила Семеновича были связаны с эллиптическими уравнениями в негладких областях. Совместно с Б. А. Амосовым он получил точные по порядку оценки сингулярных чисел для интегральных операторов типа потенциала на липшицевой поверхности. С помощью этих результатов для самосопряженных операторов на кусочно гладких поверхностях была получена асимптотика собственных значений. (Впоследствии условие кусочной гладкости было снято Г. В. Розенблюмом и Г. М. Тащияном.) Михаил Семенович исследовал спектральные свойства задач в липшицевых областях для уравнения Гельмгольца и для системы Ламе.

Совместно с Г. В. Розенблюмом Михаил Семенович изучал возникший в квантовой физике класс краевых задач для оператора Дирака в области $\Omega \subset \mathbb{R}^{3}$, особенностью которых было нарушение условия Шапиро-Лопатинского. Эта аномалия приводила к неэллиптичности оператора, возникающего при сведении задачи на границу. Тем не менее, для таких задач была установлена базисность собственных функций и найдена асимптотика собственных значений. Аналогичные результаты были получены и для оператора Максвелла.

В последние двадцать лет Михаил Семенович занимался развитием теории спектральных краевых задач при ослабленных требованиях на гладкость коэффициентов и границу области (вплоть до липшицевых границ), а также анализом задач в рамках общих банаховых функциональных пространств, таких как пространства Бесова или пространства бесселевых потенциалов. Все это потребовало привлечения ряда новых идей и технических приемов. Особый упор на липшицевы поверхности не случаен. С одной стороны, класс таких поверхностей легко и наглядно описывается, в нем все еще можно естественным образом проводить постановку краевых задач для эллиптических систем второго порядка (в классической или вариационной формулировке); при дальнейшем расширении класса поверхностей эти свойства утрачиваются. В то же время этот класс включает в себя основные естественные типы поверхностей, такие, например, как кусочно гладкие с допущением особенностей различной размерности. Наконец, анализ задач в липшицевых областях представляет собой вызов исследователю, поскольку многие фундаментальные аналитические свойства уравнений и операторов резко ухудшаются при переходе от регулярных областей к липшицевым. Сочетание этих обстоятельств вызвало появление значительного количества содержательных работ видных исследователей в данной области.

Общность рассмотрений в этой серии работ М. С. Аграновича росла со временем, начиная от систем с постоянными коэффициентами и симметричной главной частью, с дальнейшим переходом к коэрцитивным системам, далеким от симметричных, затем к коэрцитивным системам второго порядка с переменными коэффициентами и, наконец, к системам произвольного четного порядка. Помимо классических краевых задач рассматривались задачи с различными типами краевых условий на разных частях границы, а также с условиями на незамкнутых поверхностях.

Типичными вопросами, которые изучались для таких систем, являлись: корректность постановки основных краевых задач в классическом и вариационном подходах, зависимость гладкости решений от гладкости граничных функций и правой части уравнения, дискретность спектра, гладкость корневых функций, характер сходимости и суммируемости спектральных разложений в различных функциональных простран- 
ствах. Фундаментальные результаты были получены как для задач со спектральным параметром в граничном условии, в первую очередь для задач типа Пуанкаре-Стеклова, так и для задач сопряжения (трансмиссии). Для задач последнего типа исследовались вопросы регулярности и полноты корневых функций, сходимости и суммируемости спектральных разложений и, наконец, оценки и асимптотические свойства собственных значений. К этой тематике Михаил Семенович многократно возвращался в течение своей долгой математической жизни. Им разработана техника сведения таких задач к интегральному или псевдодифференциальному оператору на границе или на поверхности сопряжения, обобщающая методику, ранее использовавшуюся для уравнения Гельмгольца. В общей ситуации эллиптических систем с переменными коэффициентами явное выражение для фундаментального решения недостижимо, однако в ряде случаев удается получить о нем достаточно информации, чтобы иметь возможность оправдать оценки собственных значений и их асимптотику.

М. С. Агранович разрабатывал и иной подход, с самого начала избегающий использования фундаментального решения. Он опирается на метод Саваре (основанный на анализе сходимости разностных отношений), адаптированный к случаю липшицевых областей. Этот подход позволяет проследить за зависимостью гладкости решения от гладкости правой части или граничных функций. Метод и результаты были распространены и на системы, эллиптические по Дуглису-Ниренбергу.

Занимаясь всю жизнь анализом в гильбертовом пространстве, в последние годы Михаил Семенович распространил свои исследования на ряд важных банаховых пространств, таких как пространства Бесова и пространства бесселевых потенциалов. Для липшицевых областей и поверхностей как конструкция этих пространств, так и анализ краевых задач в них требуют привлечения принципиально новых соображений. Михаил Семенович разработал схему, основанную на сочетании вещественных и комплексных методов интерполяции, для которых исходными являются результаты типа Саваре для целых или полуцелых показателей гладкости. Решающим здесь оказывается применение результата И.Я. Шнейберга об экстраполяции свойства ограниченной обратимости оператора, действующего в интерполяционном семействе пространств. В итоге М. С. Аграновичу удалось установить разрешимость краевых задач в соответствующих банаховых пространствах с показателем суммируемости в некоторой окрестности точки 2 и показателями гладкости из некоторого интервала. Также им были получены теоремы о сходимости и суммируемости спектральных разложений.

Одним из ярких результатов М. С. Аграновича последнего времени была совместная с А. М. Селицким работа, посвященная исследованию дробных степеней несамосопряженных эллиптических операторов в липшицевых областях. Авторами были найдены новые эффективные достаточные условия, при которых проблема Като (square root Kato problem) об области определения квадратного корня максимального секториального оператора имеет положительное решение.

Здесь мы не приводим списка публикаций М. С. Аграновича. Такой список имеется на его web-странице на портале http://www.mathnet.ru.

Михаил Семенович был блестящим педагогом. Его лекции и доклады на конференциях и научных школах всегда отличались прекрасным отбором материала, четкостью изложения и неформальным объяснением ключевых идей. В 2002-2007 гг. Михаил Семенович прочитал в Независимом Московском университете несколько спецкурсов по анализу: "Обобщенные функции", "Соболевские пространства", "Эллиптические псевдодифференциальные операторы”, "Несамосопряженные операторы”. Он задумал написать серию из четырех книг по мотивам этих лекций, но суждено ему было написать только первые две. Это учебник "Обобщенные функции" (2008) и монография "Соболевские пространства, их обобщения и эллиптические задачи в областях с гладкой и липшицевой границей” (2014). Его книги адресованы как начинающим математикам (скажем, аспирантам), так и экспертам. В них доступность и просто- 
та подачи материала замечательно сочетаются с изложением глубоких современных достижений в данных областях. Педагогический талант Михаила Семеновича проявился и в научном руководстве: среди его учеников Б. А. Амосов, З. Н. Голубева, Л. С. Джанлатян, А. С. Милевский, Л. Ф. Фридлендер.

М. С. Агранович был членом редколлегии журнала "Функциональный анализ и его приложения" с момента его основания в 1967 г. и заместителем главного редактора с 1991 г. до конца своих дней. Работе в редколлегии он отдавал исключительно много энергии и сил. Также он входил в редколлегию журнала "Russian Journal of Mathematical Physics".

Михаил Семенович был счастлив в браке. Его жена Оксана Андреевна Зиза тоже была математиком. Они оба любили путешествовать, собрали большую библиотеку. Воспитали дочь. Оксана Андреевна трагически погибла 15 лет назад, и Михаилу Семеновичу пришлось учиться жить одному. Дочь Ирина, внуки Евгения и Петр, правнучка Оксана живут в Сан-Франциско. Оксана любит математику, посещает математический кружок.

Ученики, друзья и коллеги Михаила Семеновича знали его как обаятельного, интеллигентного и очень благожелательного человека. Общение с ним всегда оставляло светлый след благодаря его эрудиции, ясной и лаконичной речи, настоящей увлеченности и любви к своей профессии. Он был человеком с высоким чувством долга и ответственной гражданской позицией. Многие события, происходившие в нашей стране и в мире, не оставляли его равнодушным. Нередко он находил возможность публично высказывать свою позицию по поводу волновавших его событий.

Михаил Семенович сохранял творческую активность и интерес к жизни до последних своих дней. Все, кому посчастливилось знать Михаила Семеновича Аграновича, навсегда сохранят память об этом замечательном математике и человеке.

Б. А. Амосов, В. М. Бухитабер, М. М. Маламуд, С.П. Новиков, Г.В. Розенблюм, Т. А. Суслина, Л. Ф. Фридлендер, А. А. Шкаликов 